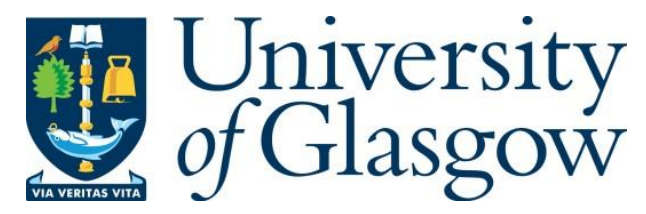

Gnani, F., Lo, K. H., Zare-Behtash, H., and Kontis, K. (2016) Shock wave diffraction in the presence of a supersonic co-flow jet. Shock Waves, 26(3), pp. 253-262. (doi:10.1007/s00193-016-0634-3)

This is the author's final accepted version.

There may be differences between this version and the published version. You are advised to consult the publisher's version if you wish to cite from it.

http://eprints.gla.ac.uk/118541/

Deposited on: 23 May 2016

Enlighten - Research publications by members of the University of Glasgow http://eprints.gla.ac.uk 


\title{
Shock Wave Diffraction in the Presence of a Supersonic Co-Flow Jet
}

\author{
F. Gnani - K. H. Lo • H. Zare-Behtash • K. Kontis
}

the date of receipt and acceptance should be inserted later

\begin{abstract}
The interaction between a diffracting shock wave and a uniform jet is a case that so far has only been partially investigated. This interaction is extremely important for the control of noise generation and improvement of combustor performance. To fill this knowledge gap, three geometries of the diffracting corner, namely a straight ramp, a serrated ramp, and a rounded corner, have been tested experimentally to study the interaction of shock diffraction with a supersonic co-flow jet at incident Mach numbers of 1.31 and 1.59, with Reynolds numbers of $1.08 \times 10^{6}$ and $1.68 \times 10^{6}$, respectively. Schlieren photography was employed to analyse the evolution of the flow phenomena. The aim is to provide a qualitative understanding of the interaction between the diffracting shock wave and the uniform jet relevant to future high-speed transport. The results show that the flow field evolves more rapidly and develops stronger structures for a higher shock Mach number. The diffraction around a rounded splitter develops a periodical vortical structure which continues after the disturbance introduced by the passage of the shock wave is removed.
\end{abstract}

Keywords Shock wave diffraction · Vortex · Schlieren · Unsteadiness

\section{Nomenclature}
$A S \quad$ Curved acoustic wave
$C S \quad$ Contact surface
$C F S L \quad$ Co-flow shear layer

F. Gnani · K. H. Lo · H. Zare-Behtash · K. Kontis University of Glasgow

School of Engineering

G12 8QQ, UK

E-mail: f.gnani.1@research.gla.ac.uk

$\begin{array}{ll}D S & \text { Diffracted shock wave } \\ E A S & \text { Expansion acoustic wave } \\ H & \text { Hump on the co-flow shear layer } \\ I & \text { Incident shock wave } \\ M & \text { Mach number } \\ O S & \text { Oblique shock wave } \\ P M F & \text { Prandtl-Meyer fan } \\ R D S & \text { Reflected diffracted shock wave } \\ R O S & \text { Reflected oblique shock wave } \\ S & \text { Stem } \\ S e a W & \text { Sea-wave shape } \\ S L & \text { Shear layer } \\ V & \text { Vortex } \\ W W & \text { Weak waves } \\ \lambda S & \text { Lambda shock }\end{array}$

\section{Introduction}

The formation of a spiral vortex from the diffraction of a shock wave over a convex corner has been extensively studied $[1-5]$. The flow features that characterise the shock wave diffraction without co-flow have been analysed with incident shock Mach numbers in the range of 1.0 to 5.0 [6]. However, the complexity of the flow scenario and the small time scales have made the capture and analysis of the processes quite challenging.

Mach numbers between 1.0 and 2.0 have acquired notable interest because the shock wave interaction with various objects in this flight regime plays an important role in aerodynamics and aero-acoustics, and must be considered in the design of the entire vehicle [7]. Two configurations are schematically illustrated in Figure 1 to show the differences in the flow pattern as the Mach number increases.

At small Mach numbers, the region perturbed by the presence of the corner exhibits small density variations. For slightly increased Mach numbers, in Fig- 
a)

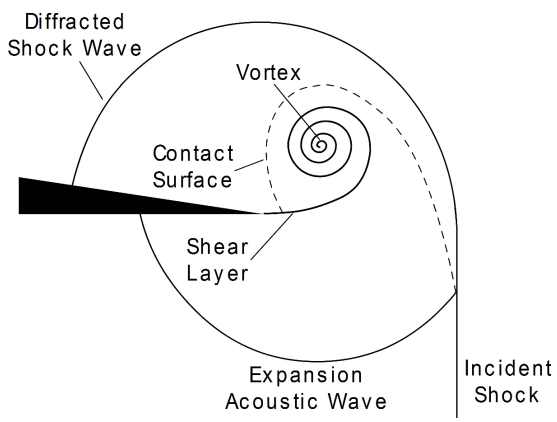

b)

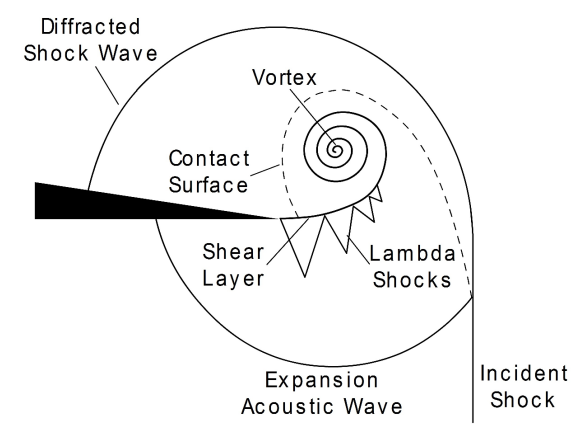

Fig. 1 Flow feature of diffraction with no co-flow around a sharp wedge at a) $\mathrm{M}=1.31 ; \mathrm{b}) \mathrm{M}=1.59$

ure 1(a), the steeper change in density and the adverse pressure gradient prevent the high-speed flow behind the shock to negotiate the corner. The detached flow that forms the slipstream winds up into a spiral vortex, which enlarges and tends to assume a more elliptical shape due to viscous dissipation [8]. It is interpreted as the separation of the boundary layer attached to the upstream wall, and thus makes a discontinuity in the tangential velocity [9]. Large gradients in density were experimentally observed by Abate \& Shyy [10] in the region occupied by the slipstream and the vortex, which is more turbulent and wider for smaller corner angles.

At a Mach number of 1.5 the characteristic lambda shock structure forms on the shear layer, schematically shown in Figure 1(b). This phenomenon, that was detected by Skews et al. [8] for an incident shock Mach number $M \geq 1.35$, becomes stronger and more visible as the shock strength increases [2]. A higher speed of the incoming gas flow means that stronger compression waves are required to slow down the flow and thus the lambda shocks tend to form a single shock. Mach numbers in the range 1.6 to 1.87 form secondary and tertiary shocks on the shear layer at the end of the lambda shocklets.

Rounded geometry profiles, schematically illustrated in Figure 2, are characterised by a zone of recompression between the main vortex and the wall. The experimental investigation conducted by Skews [11] led to the conclusion that the change in radius does not affect the overall flow pattern. For Mach numbers greater than 1.45, in Figure 2(b), the incoming flow expands, becoming locally supersonic in the vicinity of the corner, and develops lambda shocks on the shear layer.

To the authors' knowledge, not much work has been done on shock wave diffraction in the presence of a coflow jet. The majority of research on shock wave diffraction has considered the evolution of a free vortex with no shock wave interactions. The study of the interaction between a shock wave and a vortex has been motivated by the need to understand the mechanism of noise production in high-speed vehicles [12-14]. The generation of acoustic waves which grow from instabilities is a wellknown source of noise which plays an important role in aerodynamics and aero-acoustics [15].

Gongora-Orozco et al. $[16,17]$ reported that the interaction of a uniform co-flow parallel to the shock wave diffracting at a corner increases damping of the flow unsteadiness. The vortex sheet is elongated and the diffracted shock is weakened while it travels downstream. For the same shock wave Mach number of 1.66, for low co-flow Mach numbers the shock wave reflected from the test section wall is not able to pass through the vortex. A stronger co-flow, at Mach number of 0.519 , is able to penetrate the vortex. The resulting vortex/shock interaction leads to the decay of the vortex structure and the attenuation of the lambda shocklets, thus reducing the overall shock/vortex interaction.

The present study investigates the interaction of a shock wave diffracting over a ramp, a serrated, and a rounded splitter with a uniform supersonic co-flow jet of $M=1.28$. The mechanism of the formation of periodic vortical structures is examined with incident shock Mach numbers of 1.31 and 1.59. This investigation aims to provide a qualitative understanding of the interaction process of a diffracting shock wave with a uniform jet in the speed range at which aerodynamic surfaces and propulsion systems interact with the gas flow.

\section{Experimental Setup}

\subsection{Shock Tube and Test Models}

The shock tube, also employed by Gongora-Orozco et al. [18], has a square cross-section with internal dimensions of $24.8 \mathrm{~mm} \times 24.8 \mathrm{~mm}$ and a wall thickness of $2.6 \mathrm{~mm}$. The length of the driver and driven sections are $700 \mathrm{~mm}$ and $1750 \mathrm{~mm}$, respectively. The pressure in 
a)

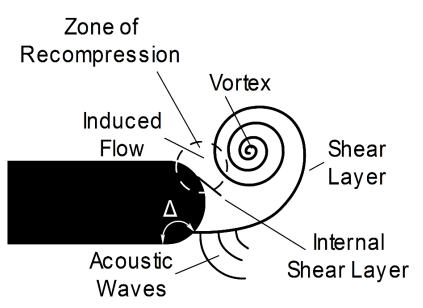

b)

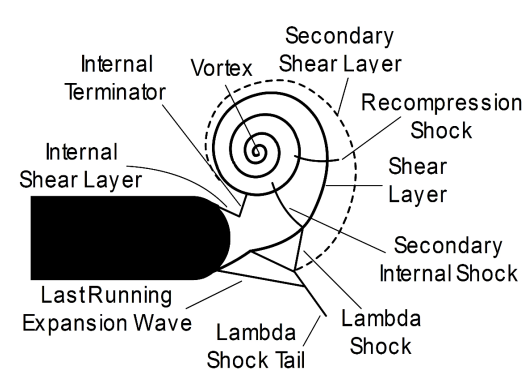

Fig. 2 Schematic of the shock wave diffraction around a curved corner for: a) $M=1.31$; $) \mathrm{M}=1.59$

the driver section was monitored using a Kulite XTL190 transducer with a range of 0 bar to 7 bar. The gas in the driven section was at ambient conditions. The two sections were initially separated by an acetate film ruptured by a spring-loaded plunger, in the way that a planar shock wave normal to the walls is produced and travels along the driven section reaching the test section. The thickness of the diaphragm used was 19 $\mu \mathrm{m}$ and $75 \mu \mathrm{m}$, in order to sustain the pressure ratio for generating the incident shock Mach number of 1.31 and 1.59, respectively. The Reynolds numbers based on the hydraulic diameter of the shock tube driven section are $1.08 \times 10^{6}$ and $1.68 \times 10^{6}$, respectively.

The details of the three test models used are given in Figure 3. Along with the conventional ramp and rounded geometries, a wedge with a serrated structure along the spanwise direction has been examined. The choice of the latter geometry was motivated by the interest in observing the effect of the presence of slits in the diffraction process. All the splitters are of the same length of $103.26 \mathrm{~mm}$ and thickness of $5.63 \mathrm{~mm}$. For the ramp and the serrated model, a $8^{\circ}$ slope starts from

a)

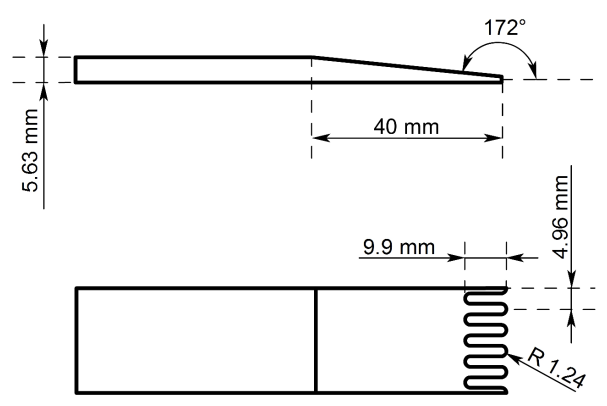

c)

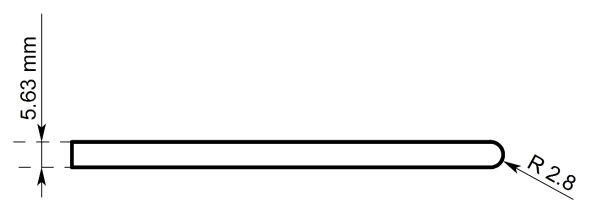

Fig. 3 Geometry details of the splitters; a) Side view of the ramp model; b) serrated model; c) Rounded model model tip and extends for a horizontal length of $40 \mathrm{~mm}$. Regarding the serrated pattern on the model tip, a distance of $2.48 \mathrm{~mm}$ was chosen to have a sufficient amount of slots in order to make the model three-dimensional and minimise the sidewall effects.

A uniform steady jet is generated as a consequence of pressure difference between a high-pressure upstream and ambient conditions and directed to the test section as illustrated in Figure 4. The co-flow channel was set before firing the shock tube for each run and was continuously fed using the inhouse supply of compressed air. It usually took under one minute between setting the co-flow pressure and firing the shock tube. At the junction with the shock tube, a supersonic nozzle designed with the method of characteristics guarantees to establish a supersonic and parallel flow in the upper half of the test section [19]. For the measurement of the co-flow jet, since the nozzle is set for a specific Mach number, the corresponding pressure ratio to achieve the nozzle Mach number was established at the two ends of the channel and kept constant throughout the entire experimental campaign. The co-flow velocity was theoretically determined by applying the two-dimensional isentropic equations for oblique shocks and expansion fans which can be found in gasdynamics books [7]. By using schlieren for the ramp case to determine the de-

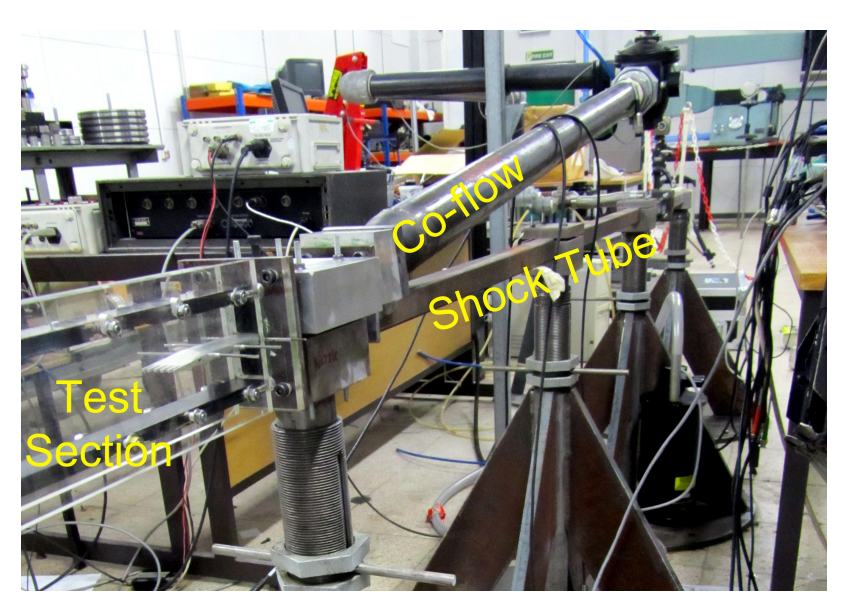

Fig. 4 Shock tube setup 
flection angles, the isentropic co-flow Mach number was found to be 1.28 , corresponding to a jet flow velocity of $380 \mathrm{~m} / \mathrm{s}$ at the atmospheric temperature of $293 \mathrm{~K}$.

\subsection{Schlieren Photography}

A Toepler Z-type schlieren photography configuration identical to that used by Zare-Behtash et al. [20] was employed in the present study. A continuous light beam emitted by a $450 \mathrm{~W}$ Xenon arc lamp passes through a condenser lens with a $79 \mathrm{~mm}$ focal length and a slit before being collimated by a parabolic mirror of $203.3 \mathrm{~mm}$ diameter and $1016 \mathrm{~mm}$ focal length. The light beam then illuminates the test section and is focused by another parabolic mirror at a knife-edge and passes through a magnification lens of $49 \mathrm{~mm}$ diameter. A Photron SA3 monochrome high-speed camera, with 12-bit dynamic range, is used to record the images at a frame rate of 16000 fps. The exposure time of the camera is set to $2 \mu \mathrm{s}$.

The acquired schlieren images were processed using ImageJ. The wind-on images captured were processed by subtracting a reference wind-off (no flow) image in order to remove non-uniform illumination and imperfections in the test section windows.

\section{Results and Discussion}

\subsection{Influence of Geometry}

\section{Ramp Geometry}

The shock wave diffraction pattern at different time instants obtained from schlieren photography experiments is analysed in this section. The reference time $t_{0}=0$ for each set of images is taken as the time immediately before the incident shock wave diffracts at the corner.

As Figure 5 illustrates, initially, before the incident shock encounters the area expansion at the corner, the co-flow jet is not subject to the influence of the moving shock wave. Due to the geometrical characteristics, in the case of the ramp, the jet expands through a PrandtlMeyer fan, $P M F$, at the first area change on the upper half of the test section. The accelerated flow reaches the tip of the splitter and, due to the pressure difference between the upper part of the model and the lower part, behaves as a half over-expanded nozzle. A straight wake, labelled co-flow shear layer, $C F S L$, is generated at the model tip. Here, also the oblique shock, $O S$, takes place, redirecting the flow parallel to the test section walls. This shock wave is reflected from the test section wall generating the reflected oblique shock, ROS.

As Figures 6 shows, once the incident shock wave, $I$, which travels normal to the test section wall, reaches the model tip, it encounters an area change and thus expands opposing the co-flow jet. In Figure 6(a), the flow features are similar to those previously described in Figure 1 , such as the diffracted shock wave, $D S$, and the expansion acoustic wave, $E A S$. This suggests that in the early stages of the interaction a vortex, $V$, appears at the model tip, confirmed by the presence of the disturbance visible from the hump, $H$, on the co-flow shear layer. This structure is generated by the interaction of the incident shock wave with the co-flow, however, the presence of the continuous jet tends to flatten the hump while it is swept downstream, leading to the formation of a sea-wave shape, SeaW in Figure 6(b). The co-flow deforms the diffracted shock which, in the part near to the test model, does not travel upstream indicating that this wave has a lower strength than the co-flow. On its curvature, the diffracted shock meets and then merges with the oblique shock, $O S$, which forms at the corner tip before the shock diffraction, as Figure 6(b) illustrates.

Furthermore, the incident shock and the diffracted shock are visibly separated in the point where, in the case without co-flow in Figure 1, the expansion acoustic wave meets the contact surface. The contact surface, defined as a surface separating the gas flow which passes through the normal shock wave from that exposed to the curved diffracted shock [11], does not occur in this case. Instead, as a consequence of the different velocity in the channels above and below the splitter, the diffracted shock is stretched. As shown in Figure 6(b), since the region perturbed by the presence of the vortex is separated to match the undisturbed condition ahead of the incident wave, the incident shock links with the diffracted shock through a small stem, $S$. It can be noted that, in Figures 6(a) and 6(b), the incident shock continues its motion beneath the co-flow shear layer towards the downstream direction and no apparent disturbance by the propagation of the expan-

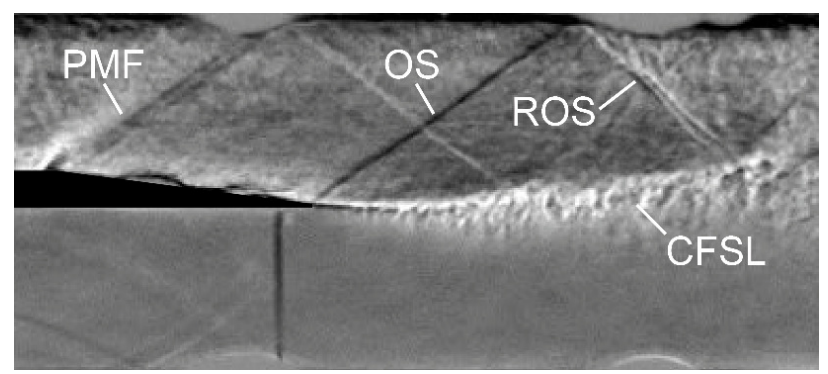

Fig. 5 Schlieren picture of the ramp corner at the time $t_{0}=0$ 


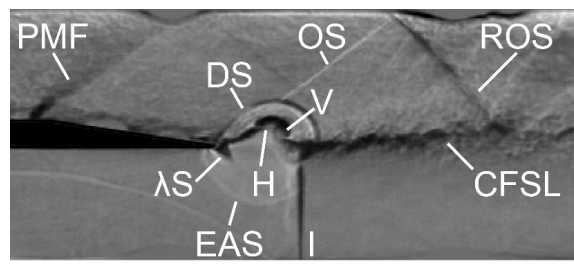

a) $t_{1}=62.5 \mu \mathrm{s}$

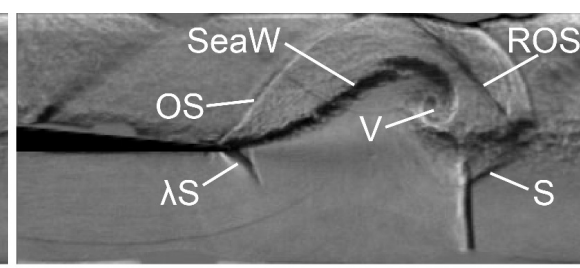

b) $t_{2}=125 \mu \mathrm{s}$

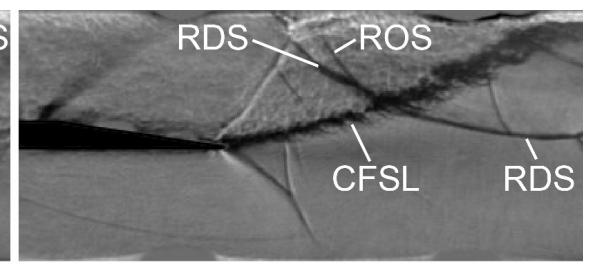

c) $t_{3}=250 \mu \mathrm{s}$

Fig. 6 Different time instants of schlieren photographs of shock wave diffraction around the ramp geometry with $M=1.59$

sion acoustic wave, $E A S$, was observed. A similar behaviour is also exhibited by the reflected oblique shock, $R O S$, after the passage of the diffracted shock wave, $D S$.

Figure 6(c) shows the flow pattern established after the diffracted wave reflects from the upper wall of the test section, called $R D S$. This shock wave travels back toward the center of the test section, passing through the reflected oblique shock, ROS. Although the vortex/shock interaction is not clear due to the limitation in image resolution, the parts of the shock wave beneath and above the co-flow shear layer remain connected while passing across it. The co-flow shear layer is a turbulent wake which does not act as a separation surface thus allowing the reflected wave to pass across it.

As expected, for this Mach number a lambda shocklet, $\lambda S$, appears on the shear layer just after the incident shock has diffracted over the splitter surface and grows in size as the time progresses, see Figure 6(b). It has been established that small lambda shocks perpendicular to the shear layer appear due to the need of the vortex-induced flow to be decelerated while moving downstream over the shear layer [8]. A lambda shock usually occurs when a normal shock wave strongly interacts with the boundary layer forming a structure with two legs which merge into a normal shock at a certain distance from a surface. The portion of air which passes through these two oblique shock legs is slowed down, but less than that which passes through a normal shock, which is decelerated to subsonic speeds. In this case the presence of lambda shocklets means that, although the flow behind the incident shock wave is subsonic, the expansion of the flow resulting from the shear layer curvature produces a local region of supersonic speed. It can be noted that the occurrence of the lambda shock is not affected by the presence of the coflow shear layer. From Skews [6], in a quiescent gas, multiple lambda shocks are observed on the shear layer for Mach numbers 1.4 to 1.5. In the present flow configuration a single lambda shock occurs, in agreement with previous findings. The presence of the co-flow modifies the shear layer, which in turn can influence the shape of the lambda shocks, although here the difference does not appear to be substantial. An overlaying schematic with the flow features developed is given in Figure 7.

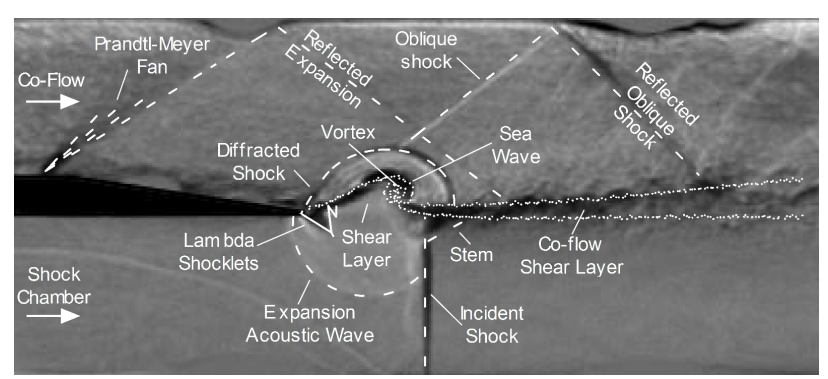

Fig. 7 Schematic of the interaction between the shock wave diffraction around a ramp geometry with $M=1.59$ and a supersonic co-flow jet. The flow is from left to right

\section{Serrated Geometry}

The flow evolution around the serrated splitter, shown in Figure 8, presents some similarities with the ramp geometry. The supersonic flow over the serrated ramp generates a co-flow shear layer, $C F S L$, similar to the one observed for the ramp geometry, except that the flow can already partially separate in the serrations. This wake also appears considerably thicker than that in the ramp geometry, as shown in Figure 8(a).

As Figure 9 illustrates the geometry of the test model produces two individual two-dimensional (2D) shock wave diffractions in different planes. These develop their own diffracted shock waves and expansion acoustic waves. The first $2 \mathrm{D}$ shock diffraction, identified as diffracted shock $1, D S 1$, and the expansion acoustic wave 1, EAS 1, in Figure 8(a), develops along the span of the model as soon as the incident shock encounters the area expansion in the internal part of the serration. As the flow navigates along the serration, it expands in two dimensions in the transverse plane at each edge of the serration. The vortical structures that develop interact amongst them generating a turbulent wake. The flow eventually arrives at the wedge extremity where another 2D shock wave diffraction occurs, which gener- 


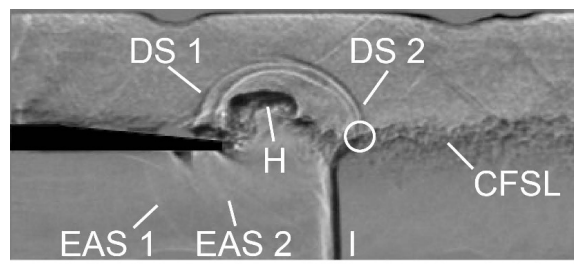

a) $\mathrm{t}_{1}=62.5 \mu \mathrm{s}$

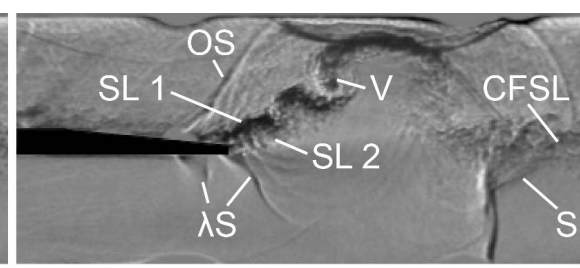

b) $\mathrm{t}_{2}=125 \mu \mathrm{s}$

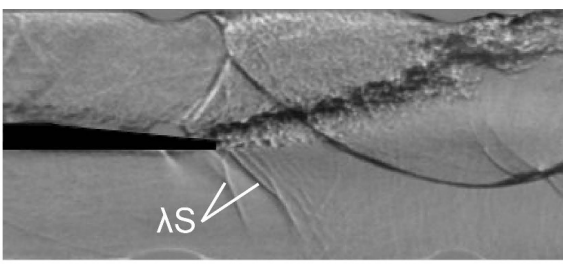

c) $t_{3}=250 \mu \mathrm{s}$

Fig. 8 Different time instants of schlieren photographs of shock wave diffraction around the serrated geometry with $M=1.59$
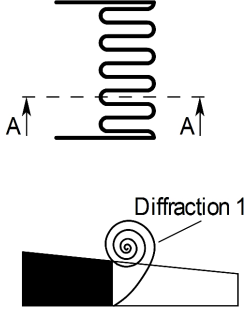

Section AA b)
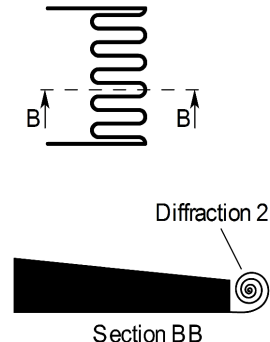

Fig. 9 a) Plane and schematic of the two-dimensional shock wave diffraction $1 ;$ b) Plane and schematic of the twodimensional shock wave diffraction 2 corresponding to the same model to Figure 8

ates the second diffraction shock, $D S 2$, and the second expansion acoustic wave, $E A S$ 2. As shown in Figure $8(\mathrm{~b})$, each of the two-dimensional diffraction processes generates a co-flow shear layer which originates from the internal part and the tips of the serration, namely shear layer $1, S L 1$, and shear layer $2, S L 2$, respectively. These two shear layers feed the wake CFSL, which has already existed prior to the shock diffraction. A schematic of the flow features which appears in this geometry is given in Figure 10.

In Figure 8(a), the jet expanding through the serrated apertures interacts with the flow from the lower side of the test section moving upwards. This interaction gives rise to the complicated shape of the hump, $H$, on the co-flow shear layer, which then develops into

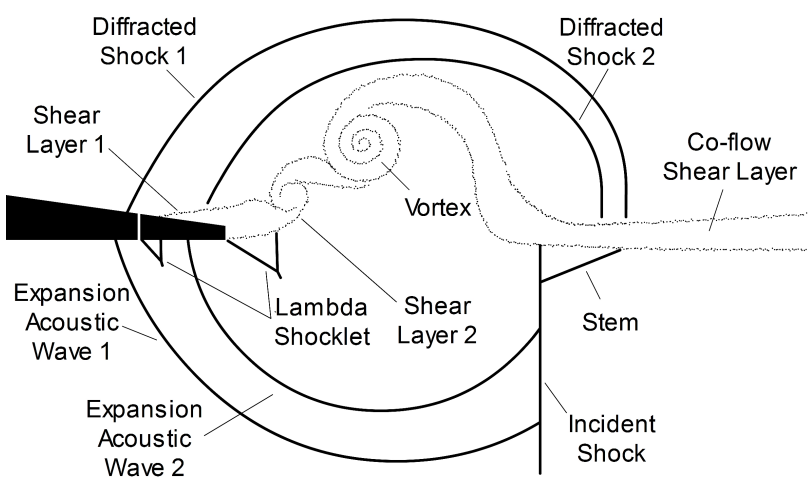

Fig. 10 Schematic of the interaction between the shock wave diffraction around a serrated splitter with $M=1.59$ and a supersonic co-flow jet

a small turbulent vortex, $V$, with a counterclockwise rotation opposing the co-flow visible in Figure 8(b). Although the vortical structure appears in the early stages of the shock diffraction, the process is deeply influenced by the presence of the co-flow jet and the vortex becomes more diffused compared to the ramp geometry previously analysed.

The diffracted waves $D S 1$ and $D S 2$ expand and meet with the incident shock, which bends passing across the co-flow shear layer, as illustrated by the circle in Figure 8(a). The stem, $S$, as previously mentioned in the ramp geometry, is completely developed in Figure 8(b). At the other end, the diffracted waves merge with the oblique shock, $O S$, originating from the internal part of the serration.

The expansion regions at the beginning and end of the serration generate two lambda shocklets, $\lambda S$, on the co-flow shear layer in Figure 8(c). In this case, since the shear layers $S L 1$ and $S L 2$ form on different planes along the spanwise direction, as the wavelets increase in size and are swept downstream, they appear overlapped.

When the diffracted shock reflects from the upper wall of the test section, in Figure 8(b), it begins to travel upstream, meets the oblique wave at the corner tip, $O S$, and interacts with the vortex. The interaction attenuates the organised structure on the co-flow shear layer, which tends to flatten towards the center of the test section and sweeps the vortex downstream.

\section{Rounded Splitter}

As illustrated in Figure 11(a), with a rounded splitter and in the absence of the incoming shock wave, the jet passes over the upper surface of the test model and smoothly expands along the curvature before separating at an angle of around $35^{\circ}$ with respect to the upper wall of the model, and forming a straight and thick coflow shear layer, $C F S L$.

Similar to the flow pattern which has been observed for the other two splitter models, shown in Figures 6 and 8 , as the diffracted shock, $D S$, expands, it becomes progressively straighter. The shape of this shock wave 
is affected by the co-flow; in Figure 11(a) it is still attached to the incident shock, $I$, but presents a discontinuity in the intersection point. Figure 11(b) shows that the diffracted shock and the incident shock propagate downstream with different velocities, similarly to the other splitter models, but the stem previously observed is not present in this case. It is believed that there is a relationship between the shear layer and the stem strength, however from the results collected here it is not possible to draw solid conclusions and further investigation is necessary.

As Figure 11(b) illustrates, the generation of the vortex, $V$, occurs when the flow on the lower channel of the test section, not able to follow the curved surface profile, detaches from it. The direction of rotation of the vortex and the formation of the hump, $H$, indicates that the incoming shock has a strength sufficiently high to go against the co-flow jet. Figures $12(\mathrm{a})$ and $12(\mathrm{~b})$ schematically show the flow evolution. A second vortex forms from the co-flow shear layer on the upper side of the model. Its generation provokes the detachment from the wall of the first one, which starts to travel downstream. A sequence of further vortices is then generated with the same mechanism, as visible in Figure 11(c). A particular feature is the appearance of a curved acoustic wave with three branches, $A S$, shown in Figure 11(b), in the lower part of the splitter tip, after the development of the vortex. As the vortex expands, this wave is scattered downstream into a series of smaller and weaker waves by the vortices which are subsequently generated from the model tip.

The symmetry of the model, along with the different flow conditions on each side produced by the different characteristics of the flows in the two channels of the test section, is believed to be the reason of the development of the structure of multiple vortices in Figure $11(\mathrm{c})$. The generation of the vortices is due to the different flow velocities in the two channels of the test section; the co-flow jet has a theoretical flow Mach number of 1.28 , and the shock interacts with it with a theoretical incident shock Mach number of 1.59. Furthermore, after the first impact the flow speed behind the incident shock drops to subsonic speeds causing a dramatic difference in the flow conditions in the two channels. The shear layer and the diffraction-induced vortex are subjected to several interactions with the diffracted shock wave reflecting from the top and bottom walls of the test section. The passage of the returning diffracted shock does not seem to produce any effect on the first vortex, but affects the subsequent vortices intensifying the break-up of the shear layer into smaller vortices.

\subsection{Influence of the Mach Number}

Figures 13(a) and 13(b) show the flow pattern around the ramp model at different incident shock Mach numbers at the same time instant of $125 \mu$ s after $t_{0}$. The figures have been chosen because the flow structures are at the same position, allowing a meaningful comparison between the two Mach numbers. A higher Mach number produces stronger interactions between the shock waves, a faster flow evolution, and the development of additional flow features. The flow is locally accelerated at the corner tip to a velocity which is high enough for the development of a lambda shock. This means that, although the flow behind the incident shock wave is subsonic, the expansion of the flow resulting from the

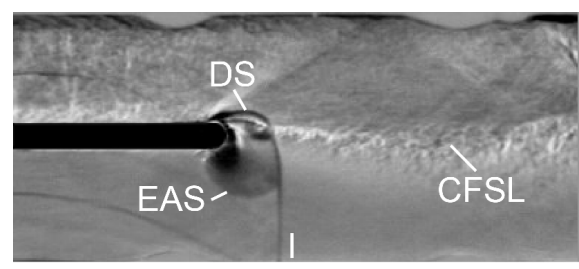

a) $t_{1}=62.5 \mu \mathrm{s}$

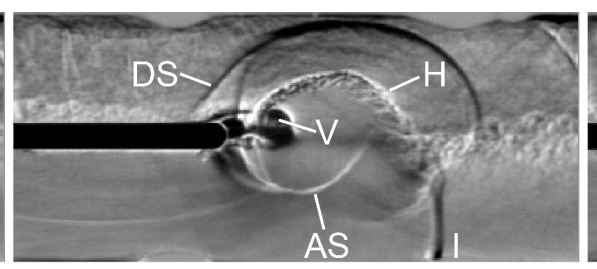

b) $t_{2}=125 \mu \mathrm{s}$

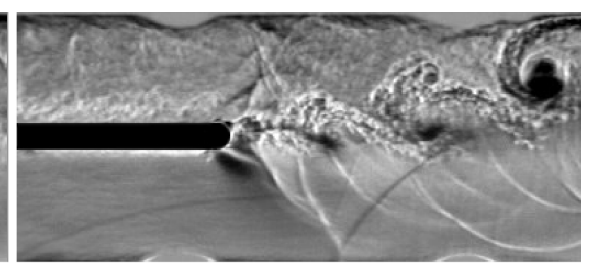

c) $t_{3}=312.5 \mu \mathrm{s}$

Fig. 11 Different time instants of schlieren photographs of shock wave diffraction around a rounded geometry with $M=1.59$
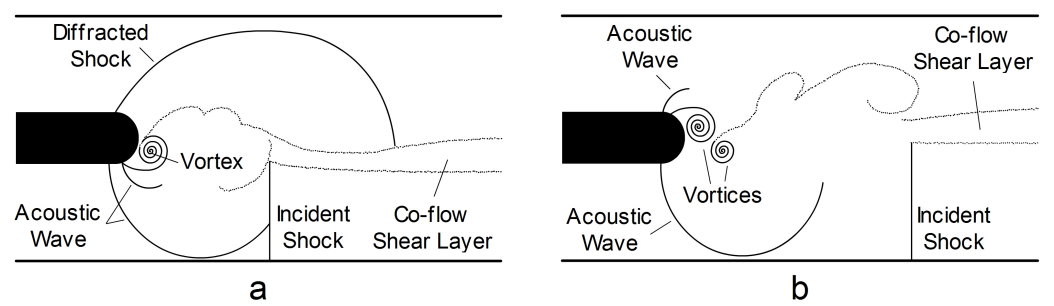

Fig. 12 Schematic of the flow evolution around a rounded geometry with a supersonic co-flow jet 


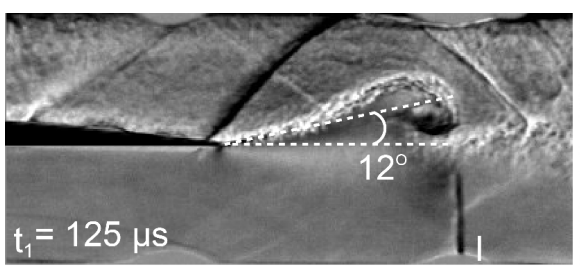

a) $M=1.31$

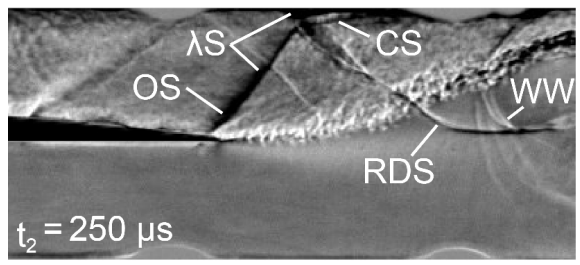

c) $M=1.31$

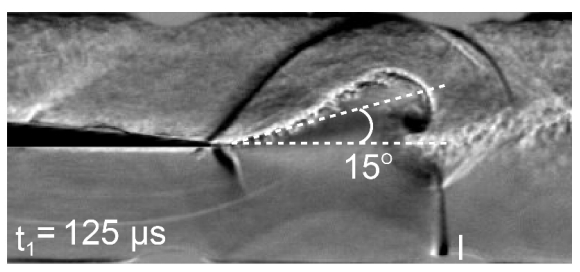

b) $M=1.59$

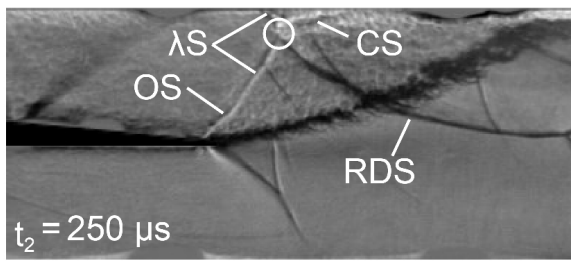

d) $M=1.59$

Fig. 13 Comparison of the flow features at different times around a ramp splitter at Mach number of 1.31 and 1.59

shear layer curvature locally accelerates the flow to a supersonic speed.

The vortex propagates downstream moving along a linear path from the model tip with a slope that increases as the incident shock Mach number increases. In Figure 13(a), for a Mach number of 1.31, the shear layer has a slope of approximately $12^{\circ}$ with respect to the horizontal, whereas in Figure 13(b), the vortex travels with an inclination of approximately $15^{\circ}$. Since the speed of the co-flow is unchanged, the vortex is swept downstream with the same velocity and, in the two images shown in Figures 13(a) and 13(b), it is at the same location along the x-axis. Additionally, the shape of the seawave appears squeezed and with a greater amplitude due to the fact that the higher Mach number of the gas flow exerts a stronger effect to oppose the co-flow jet. The fact that the vortices appear at the same position as seen in Figures 13(a) and 13(b) indicate that the stronger interaction at the higher Mach number compensates the higher vortex velocity.

Figures 13(c) and 13(d) are taken with a time step of $125 \mu$ s with respect to Figures 13(a) and 13(b), respectively. The interaction of the vortex with the diffracted shock wave, which reflects from the upper wall of the test section, labelled as $R D S$, appears to influence the vortex while travelling downstream. Figure 13(c) illustrates some weak waves, $W W$, generated from the vortex/shock interaction even though the process is not completely clear due to the limitation in the image resolution. These waves start to travel underneath the co-flow shear layer, behind the returning diffracted shock in its upstream motion, permitted by the subsonic speed of the incoming flow. The arrangement of these waves may be explained by the turbulent structure of the co-flow shear layer which causes oscillations in the spanwise direction of the returning diffracted shock, $R D S$, with a consequent overlapping of several shock waves which do not actually influence the overall flow structure.

It is observed in Figures 13(c) and 13(d) that part of the reflected diffracted shock, $R D S$, above the co-flow shear layer tends to interact with the oblique shock, $O S$, established at the model tip. The intersection of these two shock waves appears similar to the lambda shock structure which forms inside a supersonic nozzle. Two lambda shocks, $\lambda S$, are present: one on the upper test section wall and the other on the shear layer, whose tails merge in the middle of the channel, and circled in Figure 13(d). According to Babinsky [21], the flow behind the normal shock stem is subsonic whereas the flow crossing the front and rear legs of the lambda shock experiences a lower entropy increase which is translated in a different flow deceleration process. Due to the nature of the lambda shock configuration, the strength of the rear leg gradually increases from the start of the boundary-layer toward the triple point, where the flow conditions match those of a normal shock. The contact surface, $C S$, starting from the triple point of the Mach reflections is well-defined in both Mach numbers tested.

Figures 14(a) and 14(b) illustrate the comparison between the two Mach numbers at the same time instant for the case of the serrated splitter. Although the co-flow shear layer is considerably thick and bounded by two shear layers generated from two different points ( $S L 1$ and $S L 2$ ), an approximated angle of vortex propagation path has been determined. For an incident shock Mach number of 1.31, the shear layer has an angle of approximately $14^{\circ}$ with respect to the horizontal, whereas for a Mach number of 1.59 , it is approximately $24^{\circ}$. This shows that the slope of the co-flow shear layer 


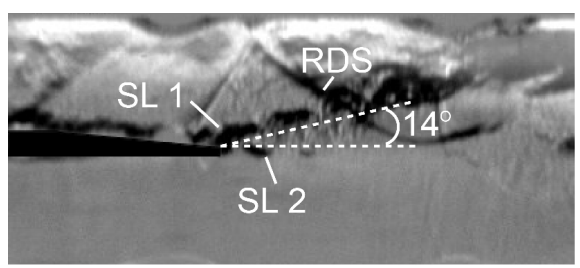

a) $M=1.31$

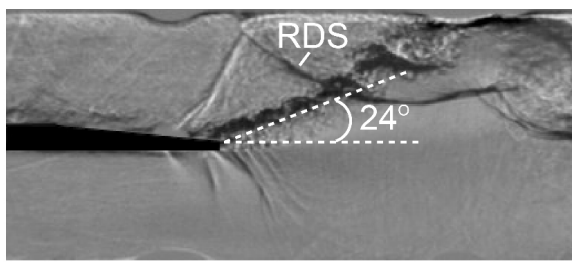

b) $M=1.59$

Fig. 14 Comparison of the flow features around a serrated splitter at Mach number of 1.31 and 1.59

depends on the strength of the incoming shock. Compared to the ramp, the increased slope of the shear layer may be due to the fact that the vortical structure forming in the inner part of the serration is protected by the vertical wall. This allows it to grow in size before the impact with the jet and to remain more compact.

Figure 14(a) also shows that on the co-flow shear layer an almost regular sequence of swirls establishes, due to the interaction of the co-flow jet with the incident shock wave. In the second case, in Figure 14(b), a more regular structure develops, at the end of which a turbulent vortex rotating in the counterclockwise direction appears on the shear layer.

In the rounded geometry, Figures 15(a) and 15(b), the generation of the first vortex appears similar in both the Mach numbers tested. In the case when the incident shock Mach number is 1.31, in Figure 15(a), the vortices, which develop horizontally on the shear layer, remain smaller in size. In Figure 15(b), the size of the first vortex is considerably bigger and the co-flow shear layer perturbation exhibits a smoother curvature. For a Mach number of 1.59, the vortex street, inclined approximately $10^{\circ}$ with respect to the centreline, produces a greater local acceleration of the flow, evidenced by the presence of a larger expansion fan on the lower side of the splitter.

Figures 15(c) and 15(d), after a time step of $62.5 \mu \mathrm{s}$, show that the vortices on the shear layer are affected by the diffracted wave reflected from the test section wall. This interaction is disruptive in nature for the lower Mach number; as the wave passes through the vortices they start to distort and become a patch of turbulence swept downstream by the co-flow shear layer. However, for the case with the higher incident shock Mach number, the shock seems to not influence the first large vortex which remains compact.

It is interesting to note that in the case of a Mach number of 1.31, alternating vortices are continuously produced from both sides of the splitter forming a symmetrical double-row wake at the centreline of the splitter. The various waves which reflect on the walls of the test section interact with the wake. This has the effect of breaking the regular pattern on the shear layer but the vortex street requires some time before stopping. The influence the shock wave produces on the vortical arrangement steadily attenuates at approximately 700 $\mu \mathrm{s}$. However, after the disturbances from reflected shock waves are terminated, the co-flow shear layer starts to develop again in a single-row street of vortices rotating in the clockwise direction, as the Figure 15(e) at the time instant $t_{3}=2875 \mu$ s illustrates. This flow structure is caused by the different velocities in the channels above and underneath of the test model.

The re-establishment of a secondary vortex street after the breakdown of the primary one is a known phenomenon investigated by Taneda [22]. In the case of the shock incident Mach number of 1.59, the two rows are switched compared to the previous case. The first three vortices in Figure 15(d) appear to have the same rotational direction with progressively decreasing strength. In this case the presence of shocks reflecting from the test section walls affects the vortex street for a longer time. In Figure 15(f), taken at the time instant $t_{4}=5875 \mu \mathrm{s}$, the co-flow shear layer starts to develop a double-row street of vortices rotating in the opposite direction at around $1300 \mu \mathrm{s}$. This pattern stops at approximately $7300 \mu \mathrm{s}$ when both the channels begin to behave as two supersonic nozzles whose jets meet in correspondence of the centreline. The different flow configuration in Figures 15(e) and 15(f) are attributed to the initial flow condition in the channel of propagation of the incident shock. The diffraction of this shock wave will produce vortices and reflected shock waves of different strength which give rise to vortex/shock interactions that produce a dissimilar flow pattern.

\section{Conclusions}

A qualitative analysis has been conducted to study the mechanisms of the shock wave diffraction with three different splitter models with two incident shock Mach numbers.

The initial undisturbed flow patterns change when the incident shock wave arrives at the tip of the test model where it diffracts. The vortex with the same flow features reported in literature with no co-flow seems to 


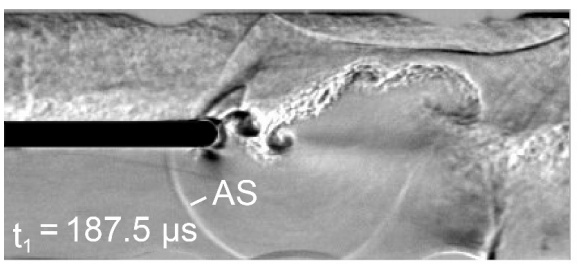

a) $M=1.31$

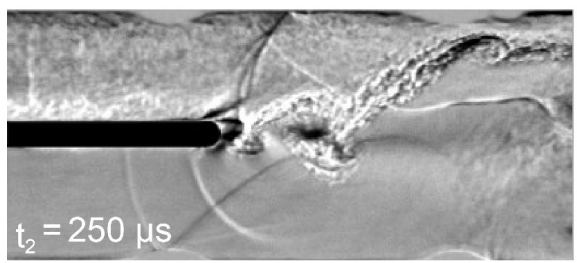

c) $M=1.31$

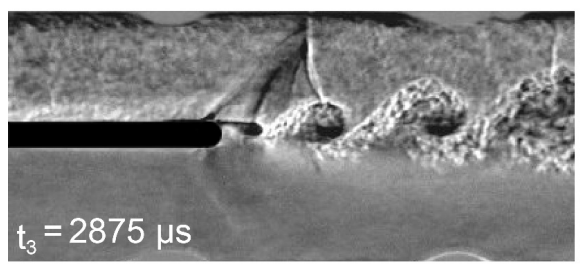

e) $M=1.31$

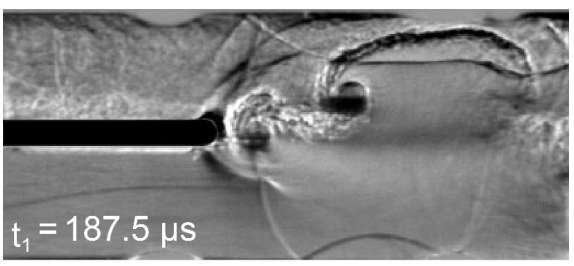

b) $M=1.59$

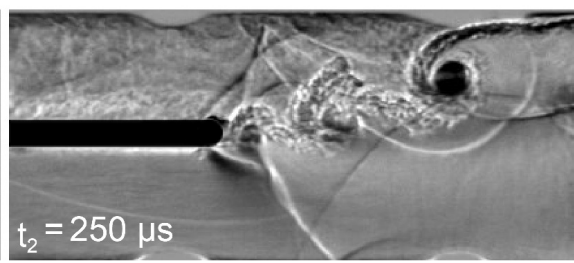

d) $M=1.59$

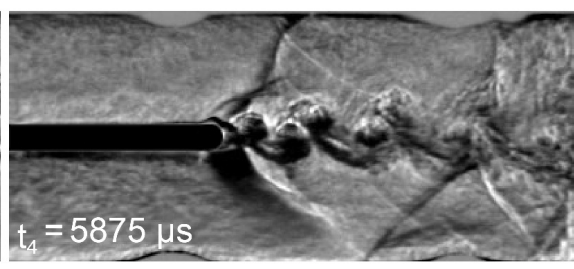

f) $M=1.59$

Fig. 15 Comparison of the flow features at different times around a rounded splitter at Mach number of 1.31 and 1.59

appear in the initial stages. Nonetheless, the presence of the co-flow propagating in the same direction of the incident shock introduces a disturbance which affects the diffracted shock evolution as the jet opposes the growth of the vortex. The diffracted shock is stretched horizontally by the co-flow and a gradual bending of the incident shock occurs in the rounded model. In the ramp and serrated splitters a stem develops on the incident shock in order to balance the flow conditions of the undisturbed region ahead of the incident shock.

The flow with the highest Mach number causes the co-flow shear layer to be more inclined with respect to the horizontal. This generates local regions of supersonic flow and lambda shock structures.

The rounded splitter gives rise the development of a series of periodic small vortices on the shear layer. Different wake configurations have been observed behind the first vortex in the two Mach numbers tested. After the effects of shock reflection on the test section surfaces are attenuated, a secondary vortex street establishes. Care should be taken during analysis of the findings due to the sensitivity of the flow structure to the current experimental setup.

The small dimensions of the geometry and the limited resolution of the schlieren images did not allow to completely clarify the evolution of the vortex/shock interaction but allowed to identify the large-scale flow features. Further investigation with a higher frame rate and a closer examination window, particularly in the early stages of the phenomena, is necessary. Additionally the employment of quantitative methodologies and optical access from above and/or underneaths the test section will be useful.

Acknowledgements The authors are indebted to the EPSRC Engineering Instrument Pool, especially Mr. Adrian Walker for the loan of the high-speed camera.

\section{References}

1. Bazhenova, T.V., Gvozdeva, L.G., Nettleton, M.A., "Unsteady interactions of shock waves," Progress in Aerospace Sciences 21(4), 249-331, (1984).

2. Skews, B.W., "The shape of a diffracting shock wave," Journal of Fluid Mechanics 29(2), 297-304, (1967).

3. Whitham, G.B., "New approach to problems of shock dynamics, Part II: three-dimensional problems," Journal of Fluid Mechanics 5(3), 369-386, (1959).

4. Bazhenova, T.V., Gvozdeva, L.G., Zhilin, Yu.V., "Change in the shape of the diffracting shock wave at a convex corner," Acta Astronautica 6, 401-412, (1977).

5. Lighthill, M.J.,"The diffraction of blast. I," Proceedings of the Royal Society of London. Series A: Mathematical and Physical Sciences 198, 454-470, (1949).

6. Skews, B.W., "The perturbed region behind a diffracting shock wave," Journal of Fluid Mechanics 29(4), 705-719, (1967). 
7. Anderson, J.D., "Modern compressible flow, with historical perspective," McGraw-Hill, 2nd edition, 1990

8. Skews, B.W., Law, C., Muritala, A., Bode, S., "Shear layer behavior resulting from shock wave diffraction," Experiments in Fluids 52(2), 417-424, (2012).

9. Sun, M., Takayama, K.,"The formation of a secondary sock wave behind a shock wave diffracting at a convex corner," Shock Waves 7, 287-295, (1997).

10. Abate, G., Shyy, W., "Dynamic Structure of Confined Shocks Undergoing Sudden Expansion," Progress in Aerospace Science 38(1), 23-42, (2002).

11. Skews, B.W., "Shock wave diffraction on multi-facetted and curved walls," Shock Waves 14(3), 137-146, (2005).

12. Dosanjh, D.S., Weeks, T.M., "Interaction of a starting vortex as well as a vortex street with a traveling shock wave," AIAA Journal 3(2), 216-223, (1965).

13. Chang, S.M., Chang, K.S., Lee, S., "Reflection and penetration of a shock wave interacting with a starting vortex," Physics of Fluids 42(4), 796-805, (2004).

14. Jones, D.S., "The scattering of sound by a vortex sheet," Journal of the Institute of Mathematics and Its Applications 15(1), 33-57, (1975).

15. Barbosa, F.J., Skews, B.W., "Shock wave interaction with a spiral vortex," Physics of Fluids 13, 3049-3060, (2001).

16. Gongora-Orozco, N., Zare-Behtash, H., Kontis, K., "Particle Image Velocimetry Studies on Shock Wave Diffraction with Freestream Flow," 48th AIAA Aerospace Sciences Meeting Including The New Horizons Forum and Aerospace Exposition, Orlando FL, USA, AIAA 2010-876, (2010).

17. Gongora-Orozco, N., Zare-Behtash, H., Kontis, K., Hale, C. , "Particle image velocimetry studies on shock wave diffraction with co-flow jet," 14th International Symposium on Flow Visualization, Daegu, Korea, (2010).

18. Gongora-Orozco, N., Zare-Behtash, H., Kontis, K., "Global unsteady pressure-sensitive paint measurements of a moving shock wave using thin-layer chromatography," Measurement 43(1), 152-155, (2010).

19. Quinn, M.K., "Shock diffraction phenomena and their measurement," PhD thesis, University of Manchester, (2013).

20. Zare-Behtash, H., Kontis, K., Gongora Orozco, N., Takayama, K., "Shock wave-induced vortex loops emanating from nozzles with singular corners," Experiments in fluids 49(5), 1005-1019, (2010).

21. Babinsky, H., Harvey, J.K., "Shock Wave-BoundaryLayer Interactions," Cambridge University Press, 2011

22. Taneda, S., "Downstream development of the wakes behind cylinders," Journal of the Physical Society of Japan 14, 843-848, (1959). 